RESEARCH NOTE

\section{Montenegro Skin Test - Evaluation of the Composition and Stability of the Antigen Preparation}

\section{Carlos Alberto da Costa $/{ }^{+}$, Vicente de Paulo Coelho Peixoto de Toledo, Odair Genaro*, Paul Williams*, Wilson Mayrink*}

Departamento de Análises Clínicas e Toxicológicas, Faculdade de Farmácia *Departamento de Parasitologia, Instituto de Ciências Biológicas, Universidade Federal de Minas Gerais, Caixa Postal 486, 31270-901 Belo Horizonte, MG, Brasil

Key words: Montenegro skin test - antigen - stability

Skin hypersensitivity tests are used for the diagnosis of various diseases. In cutaneous leishmaniasis, the Montenegro skin test ( $\mathrm{J}$ Montenegro 1926 Ann Fac Med Univ São Paulo 1: 323-330), has been used on a large scale in the New World. The test becomes positive a few weeks after infection, and it has been used for differential diagnosis of deep dermatomycosis. The Montenegro skin test is a decisive method for the diagnosis of older leishmanial lesions and mucosal lesions, when the number of parasites is low and therefore, difficult to detect. The test is also useful for follow-up in vaccination programs (W Mayrink et al. 1979 Trans $R$ Soc Trop Med Hyg 73: 385-387), and as a parameter for evaluation and of the development of immune protection (CMF Antunes et al. 1986 Int J Epidemiol 15: 572580).

Despite its use for many years, some points have been constantly questioned, one of which refers to the antigen composition when the majority of laboratories use complex parasite extracts (PEC Manson-Bahr et al. 1959 Trans $R$ Soc Trop Med

\footnotetext{
This work was supported by PRPq/UFMG, CNPq, FAPEMIG, Fundação Nacional de Saúde and Prefeitura Municipal de Caratinga.

${ }^{+}$Corresponding author. Fax: 55-31-441.6909

Received 21 June 1995

Accepted 29 January 1996
}

Hyg 53: 380-383, MCS Guimarães et al. 1974 Rev Inst Med Trop São Paulo 16: 145-148, JJ Shaw \& R Lainson 1975 Trans $R$ Soc Trop Med Hyg 69: 323-335, RA Neal \& RA Miles 1976 J Trop Med Hyg 79: 32-37, SG Reed et al. 1986 Am J Trop Med Hyg 35: 79-85). Another point refers to the stability of the antigen preparation used, since these antigens are stocked for long periods, specially in epidemiologic surveys, and protein degradation of leishmanial antigenic preparations have been reported (R Badaró et al. 1990 Trans $R$ Soc Trop Med Hyg 84: 226-227).

In Brazil, the most used antigen to estimate the cutaneous hypersensibility during the Leishmania infection has been produced in Instituto de Ciências Biológicas - Universidade Federal de Minas Gerais (ICB-UFMG), employing the methodology standardized by MN Melo et al. (1977 Rev Inst Med Trop São Paulo 19: 161-164). However, it was made of promastigote mixture of $L$. (Leishmania) mexicana, L. (Leishmania) amazonensis and $L$. (Viannia) guyanensis. Its stability has not yet been evaluated.

This work aims at contributing to the standardization of antigenic extracts used for the Montenegro skin test. We carried out a comparative study on proved cutaneous leishmaniasis patients using either the multiple strain antigen of ICB-UFMG and a single strain antigen produced in a similar way but stocked for different periods of time. The single strain antigen was prepared from $L$. amazonensis promastigotes (IFLA/BR/67/ PH8) grown in LIT culture medium for seven days (EP Camargo 1964 Rev Inst Med Trop São Paulo 6: 13-100). Parasites were washed three times with saline, resuspended in saline containing $1 / 10000$ merthiolate and sonicated. The concentration was adjusted to $40 \mu \mathrm{g}$ of total nitrogen per $\mathrm{ml}$, as described by Melo (loc. cit.). The stability studies were performed with antigen stored for different time periods. A group of 24 patients with cutaneous leishmaniasis was injected in the forearms with the following antigenic preparations: $\mathrm{Ag} 1$ : single strain antigen stored for 14 months at $4^{\circ} \mathrm{C}, \mathrm{Ag} 2$ : freshly prepared single strain antigen, $\mathrm{Ag} 3$ : freshly prepared single strain antigen autoclaved at $120^{\circ} \mathrm{C}$ for $20 \mathrm{~min}, \mathrm{Ag} 4$ : single strain antigen stored for three months at $4{ }^{\circ} \mathrm{C}, \mathrm{Ag} 5$ : freshly prepared multiple strain. All patients also received $0.1 \mathrm{ml}$ of diluent as control for non-specific reactions. Readings were made after $48 \mathrm{hr}$, and results were expressed as the mean of two perpendicular diameters, in mm.

Twenty-two $(91.6 \%)$ of the 24 patients were positive for the five different antigens. The average nodule sizes observed were: $\operatorname{Ag} 1(21,6 \pm 13$ $5,8), \operatorname{Ag} 2(20,5 \pm 4,6), \operatorname{Ag} 3(21,1 \pm 5,4), \operatorname{Ag} 4(22,5$ 
$\pm 6,1)$ and $\operatorname{Ag} 5(22,3 \pm 5,6)$. No significant differences among the antigenic preparations were detected. None of the patients presented non-specific reactions to the diluent. The two patients that did not react to the antigens presented recent lesions, with a clinical evolution of less than 30 days.

Our results with patient carriers of tegumentar leishmaniasis in activity sustain the data of W Mayrink et al. 1993 Mem Inst Oswaldo Cruz 88 (suppl): 228, employing a clone of L. (Leishmania) amazonensis. Similar results were also obtained by MCA Marzochi et al. 1995 Rev Soc Bras Med Trop 28 (suppl): 135, when they studied patients without symptoms of cutaneous leishmaniasis.

The different antigen preparations were submitted to polyacrylamide gel electrophoresis followed by silver staining (Fig.). Qualitative and quantitative differences among the profiles were found. These differences were more evident in $\mathrm{Ag} 1$ (14 months after prepared) and Ag3 (subjected to autoclaving); these profiles have fewer bands than seen in recently prepared antigen $(\mathrm{Ag} 2)$.

Protein degradation, in both cases, was evident, despite the use of merthiolate, a well known inhibitor of proteases present in promastigote extracts. Similar data were obtained with a soluble antigenic preparation used for skin tests for visceral leishmaniasis stored for different periods of time (Badaró loc. cit.).

Despite of the great advances of molecular biology, the production of molecularly defined skin test antigens is not yet possible. The identification of the components responsible for antigenic activity is undoubtly the starting point to reach this goal. Therefore, we believe our results are useful since they indicate that elements responsible for antigenic activity are present in different Leishmania strains, and that there is no need to work with complex extracts from more than one strain. Moreover, the maintenance of biologically active antigenic extracts for a long time, in spite of intense proteolytic

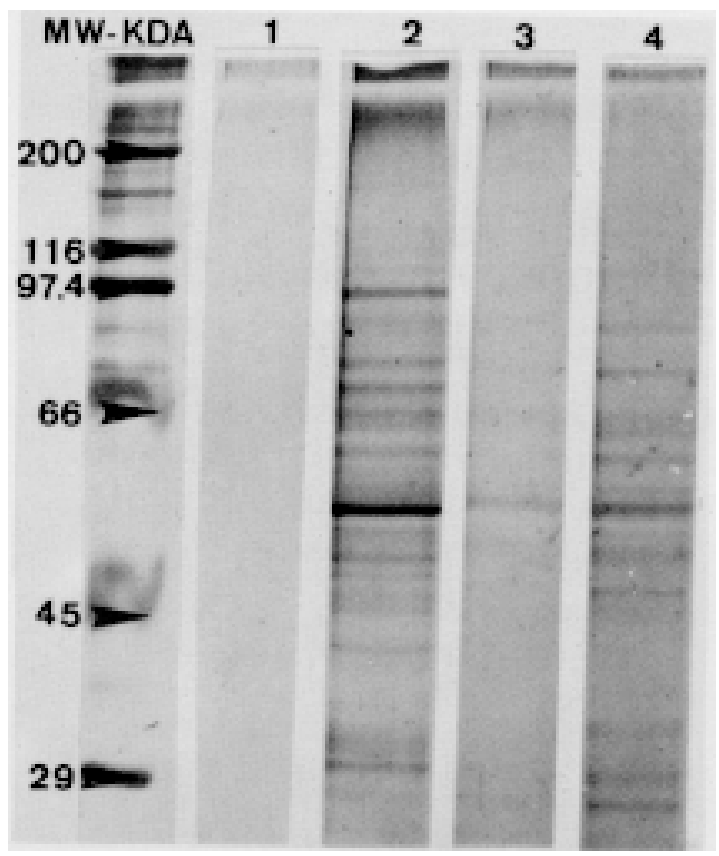

Polyacrylamide gel electrophoresis (10\% gel) of the single strain skin test antigen preparations, stained by silver nitrate. Lane 1, antigen prepared 14 months before, lane 2, antigen freshly prepared, lane 3 , antigen freshly prepared and autoclaved, lane 4, antigen prepared 3 months before.

activity or even the drastic treatment by autoclaving, suggests the possibility that elements responsible for antigenic activity are not protein or, in case they are, they would act as small peptidic units. Roberto Naiffi, from the Instituto Nacional de Pesquisas da Amazônia, described a great reactivity of the Montenegro antigen ICB-UFMG in patients with American cutaneous leishmaniasis, caused by L. guyanensis in Manaus, Brazil, even after 10 years of storage at $4^{\circ} \mathrm{C}$ (personal communication). These data are in accordance with Badaró observations (loc. cit.) with L. chagasi. 